\title{
Removal of Specular Reflection on Handheld Camera Devices
}

\author{
R.PRATHIPA ${ }^{\mathrm{a}, 1}$, M.PREMKUMAR $^{\mathrm{b}}$, M.ARUN $^{\mathrm{c}}$, S.SATHIYA PRIYA ${ }^{\mathrm{b}}$ \\ ${ }^{a}$ Associate Professor, ${ }^{b}$ Professor, ${ }^{c}$ Assistant Professor \\ Department of Electronics and Communication Engineering, \\ Panimalar Institute of Technology, Chennai, TN, India
}

\begin{abstract}
Handheld mobile devices are a dime a dozen in the industry, thanks to rapid technological advancements. However, none of the algorithms currently available on the market can remove reflections in a hand-held camera system in real time. Our Algorithm is intended to mitigate this loop as it is designed especially for smart phones, we're taking two photos of the same goal here. One shot will be taken with the flashlight turned OFF, while the other will be taken with the flash turned ON. After that, we'll perform a simple pre-processing phase called aligning the image, which involves extracting features. We will eventually get the final desired picture of good quality after all the planned operations are completed.
\end{abstract}

Keywords. Features Extraction, SIFT, RANSAC, Color Transformation, Image splicing.

\section{Introduction}

Although it is the industry standard to remove specular reflection with several flashes, our aim is to remove the castback with just two pictures. [1]. A multiple flash camera is shown in figure 1. By optimising the process, the information required to perform the scuttle can be obtained by examining just two images instead of several. The product of the illumination's spectral energy distribution and the surface reflectance is used to compute the spectral energy distribution of light reflected from an object.

Based on Dichromatic Reflection Model, the reflected light can be split into two parts because of specular and diffuse reflections. [8]. Because specular reflection is unavoidable in the real world and captures valuable scene details such as surface shape and source characteristics, the study strictly adheres to the use of specularity regions. [9]. Earlier methods for segregating reflection components based on the quantity of images used can be divided into two groups. The first group employs many images, while the others employ a limited number of images.

Many algorithms have been created to achieve this aim. These algorithms can be classified as either Lambertian assumption-based algorithm [2] or color-based algorithm or polarization-based algorithm or ICA (Independent Components Analysis) based algorithm.

${ }^{1}$ R.PRATHIPA, Department of Electronics and Communication Engineering Panimalar Institute of Technology, Chennai, TN, India; Email:prathipa.srini@gmail.com. 
For instance, ICA-based reflection removal algorithm [3], requires images obtained from a linear polarizer at various angles and these pictures must be linear combination of the original light and the reflected lights. Principle Component Analysis is used to obtain information about original light [4]. Reflection removal is also required in camera-equipped smart phones. When we try to capture image of objects behind windows, landscapes behind glass covers, or information on monitors with our phones, the reflections often extremely degrade the quality of the image and we will not be able to use it. Worse, none of the algorithms mentioned above can be used to solve the problem. The knowledge available to the algorithms processing a single image is insufficient to recreate the image behind the glass. The task of taking photos with different polarizations is impractical with a mobile.
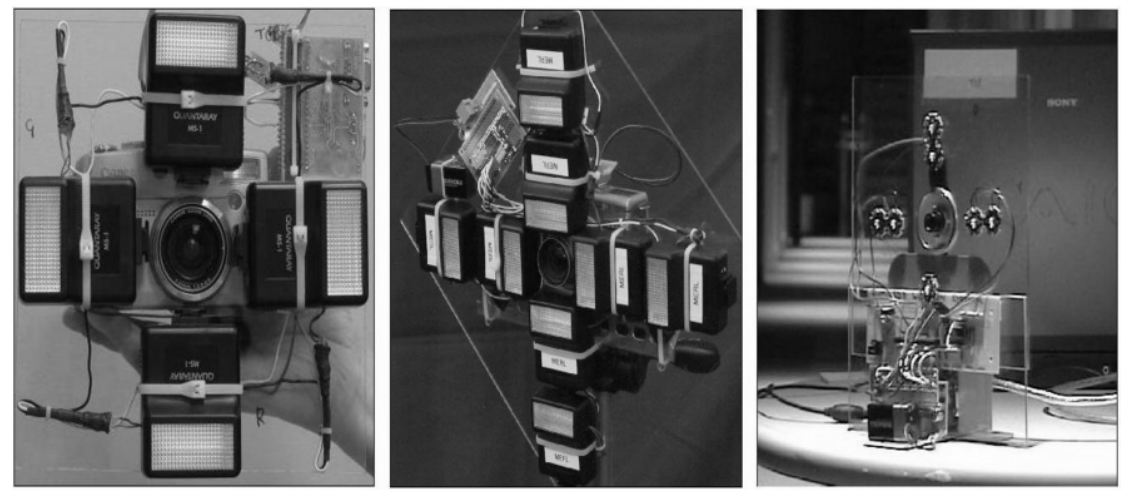

Figure 1. Multiple Flash Camera

It's also not feasible to request users to take images of the object from various angles. Here, we are introducing a reflection removal algorithm designed for mobile with camera. This algorithm requires two pictures - one should be taken with the flashlight and the other without the flashlight. After obtaining the two pictures, the proposed algorithm merges them to produce a new picture with minimal reflections. There is no restriction about the position in which the pictures are taken. It can be obtained from same or at different angle. Researches in the field of Digital Image Processing always aims to enhance the quality of the image. Whenever it comes to cast back removal or specular reflection removal denoising through multiple snaps of images is the most recent development, which is widely known by all. Other than this technique, colour based reflection removal, polarisation-based reflection removal [7] and Independent Component Analysis [10] Techniques are very popular for reflection removal [11]. Not just the field of engineering but, even medicinal field requires Digital Image Processing for inspecting and analysing certain vital internal organs through catheter imaging. The main drawback of the tongue image analysis through total variation-based image inpainting is the texture replacement, which becomes tedious. The reason being, the authors have resorted to converting the RGB image in the processing to indexed image. To do this they follow minimum variance quantization and image dithering techniques. In doing so, the space and color resolution gets hampered. But we have penned down a mitigation strategy for this problem by converting the RGB image to a gray scale image. So, the hue and saturation get removed but luminescence is still retained. We will just obtain two snaps of images in our proposal. First one will be with the 
flashlight OFF and second one will be with the flashlight ON. The one without the flashlight will only have a poor image quality. Second one will have a very good light quality but, the undesired flash will be present. So, we just replace the information lost due to the flash with the information from the First image. The user must click once and the app will capture two pictures consecutively, with the flashlight on for the second picture alone. This algorithm has a sequence of operation as shown in Figure 2.

The second part will examine the features of the two images obtained and clarify our algorithm's overall concept and sequence. Third part describes how to recognise and identify the flash spot in the photograph taken with the flash turned on. The fourth segment demonstrates how to transform the colour of one source image to reduce the impact of different lightings. The fifth section discusses the process of combining the two pre-processed source images to create a natural-looking image. Finally, the sixth segment will summarise the algorithm's benefits and drawbacks.

\section{Sequence of Operation}

The sequence begins with the image acquisition process, which is done twice, once with the flashlight turned off and once with the flashlight turned on. These two images will provide the necessary amount of data to identify and remove unwanted information. Then, using the SIFT and RANSAC algorithms, features are extracted. After studying the characters, we move on to the crucial portion of colour transformation, which involves copying the weighted average value of nearby pixels using several reference points. Finally, the skewed information from the flash is softly combined with the weighted average value.

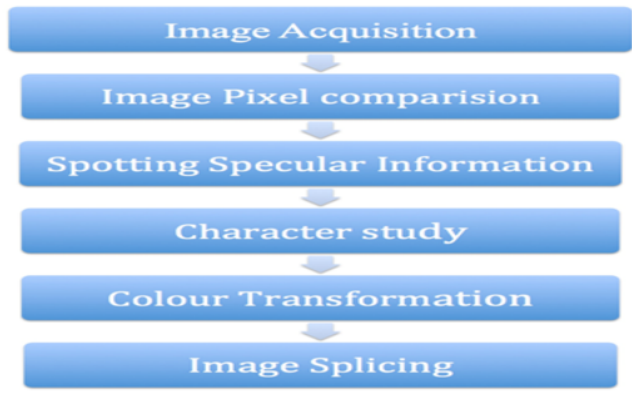

Figure 2. Flowchart of Sequence Operation

The example of two input pictures for the algorithm is shown in the diagram below. The two photos were taken at night in an indoor setting from two slightly different angles. The algorithm could have a wide range of applications in medicine [5]. Figure 3 shows a photograph taken without the use of a flash. As can be seen, the glass has a lot of reflections on it, which seriously reduced the image quality. Figure 4 shows a photo taken with the flash turned on. First, we must determine the location and magnitude of the flash spot in the photograph. Second, since the two input images may be taken from different positions, alignment of pictures should be done. Third, the two images have colour inconsistencies due to the different light sources. Before merging the two images, we must first change the colour of one to match the colour of the other. Finally, combining the flash-spot region of Figure 3 with the rest of Figure 4 results in an artificial image. To get a natural-looking result, we'd have to find out how to softcombine the two images. 


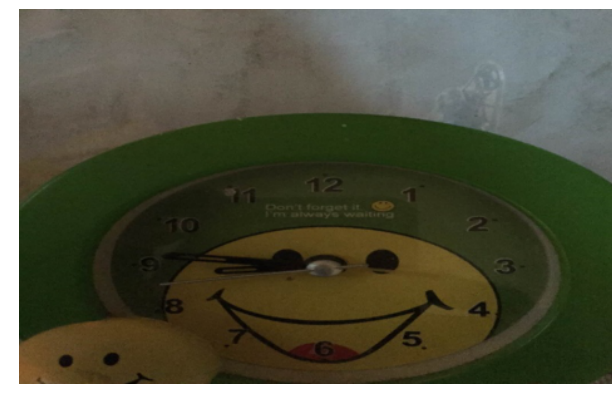

Figure 3. Picture without Flashlight

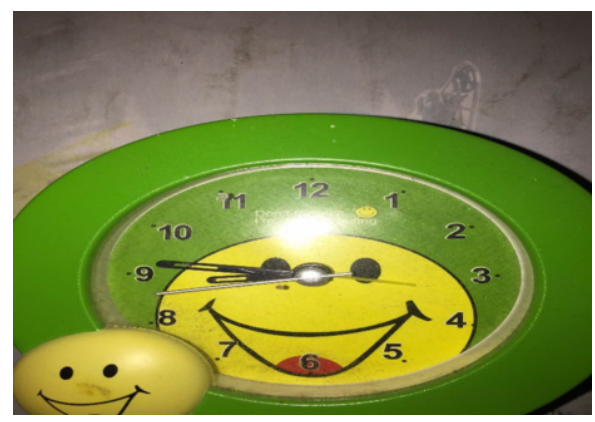

Figure 4. Picture with Flashlight

\section{Flashspot Analysis}

The goal now is to use the information from figure 3 to replace the affected region in figure 4 . We will need to locate the location and size of the flash spot in figure 4 to accomplish this.

\subsection{Identification of Flashspot using Matched Filter}

As we have seen many photos with flash spots, we can understand that the flash spots have a particular pattern - that is, they are very bright in the middle and fade away when you move away from it. This resemblance indicates that using a matched filter to locate the flash spot in a photograph taken with the flash turned on is a smart idea. We were able to conclude that using a Gaussian Filter is the best way to get rid of this castback by analysing the flash points.

The following question is how we determine the size of this Gaussian filter. Since we can't tell the extent of the flash spot ahead of time, it's unlikely that we will be able to find a Guassian filter that suits it. However, we discovered through experiments that constraining the sigma of the Gaussian filter to $1 / 200$ to $1 / 20$ of the width of the picture has no effect on the matching result in real time applications. Therefore, we simply set sigma $=(1 / 100) *$ Width in our algorithm.

\subsection{Flashspot Estimation}

After you've figured out where the flash spot's core is, you'll need to figure out how big it is. The diameter we're talking about is the equivalent diameter of the flash spot, which diminishes from its middle slowly. We binarize the image with a threshold of 0.85 to exclude most of the bright areas in the image, since the flash spot is very bright in the image. Then, we obtain the binarized image's connected components and select the components that contains the flash spot centre which we got earlier. Finally, we can estimate the size of the flash spot by calculating the equivalent diameter $\mathrm{d}$ of this identified connected part. 


\section{SIFT}

As SIFT can find distinguishing key points which are constant to spot, size and rotation, and strong to affine transformations, as well as variations in lighting, it is useful for object identification. The algorithm extracts SIFT features from the source image. These characteristics are compared to the SIFT function database created from the training images. Even though the distance ratio test mentioned above eliminates several false matches caused by background clutter, there are still matches that belong to different items.

\section{Color Transformation}

Our primary goal is to ensure that the original is improved while remaining normal [6]. In this case, colour transformation is extremely important. We've decided to remove the details from the second image's flash site and replace it with data from the first figure, which was taken without the flashlights turned ON. This can seem to be child's play at first, but it really works. We also try to increase the number of reference points from which we calculate the value of pixels in the target region. In addition to the colour space size, there are two other considerations to consider when locating neighbour pixels. The first consideration is the distance between the neighbouring pixel and the original point on the picture. To reduce the impact of irregular illumination in the frame, we'd like to highlight the points that are near to the original point. The distance between the neighbour point and the flash spot centre is the second element. We want the neighbour points to be far away from the flash point core because the flash point is the undesirable information in Figure 4.

\section{Image Splicing}

Finally, we are going to see how to combine the two images together. As we mentioned earlier, the only area which we don't require in figure 4 is the flash point i.e the bright spot. So, our idea is to use the flash point area of Figure 3 in Figure 4 to overcome the bright spot. So, with the help of Gaussian mask, we can get the combined image.

\section{Results}

In this paper we have discussed the pipeline of proposed algorithm. Initially, we find the position and extent of the flash point in the image with the help of template matching and the connected component method. Then the images are aligned by extracting the features and matching it. Next, we resolve the color inconsistency in the two pictures due to difference in the light source. At last, we combine both images in order to get the resultant image with maximum reflections removed. But still there is scope for improvement in Digital Image Processing methods. Whatever is the extent to which an algorithm is said to remove the reflections reaching ideal result is a far cry. However, by experimenting. We understood that in real time applications, if we set sigma value of Gaussian filter between 0.005 to 0.05 of the width of the image, it doesnt have impact on the matching result. Therefore, here we use sigma as 0.01 of width of picture. As we have already discussed through the course of our project, two images can also be taken from any angle. As the images may be of different angles, aligning them before combining is must. We have used standard algorithms like SIFT 
descriptors and RANSC with holography model. Both SIFT and RANSAC best serves the purpose. Thus, we can only conclude that out of all the novel techniques available, cast back scuttle on handheld device produces the best results.

\section{Conclusion}

Thus, the process of cast back scuttle has been divided into various stages and each segment is dealt with individually. First the image is obtained, then it is aligned, followed by reading in flash image, converting it into a binary image, and once we convert it to a binary image, we resort to creating a Gaussian mask to perform colour transformation to do reflection removal and eventually combine the images to obtain the processed or desired image.

\section{References}

[1] Tsuji, T., \& Inoda, R. (2010). Removal of Specular Reflection Based on High-Speed Camera Images. IEEJ Transactions on Industry Applications, 130(3), 261-267. https://doi.org/10.1541/ieejias.130.261

[2] Basri, R., \& Jacobs, D. W. (2003). Lambertian reflectance and linear subspaces. IEEE Transactions on Pattern Analysis and Machine Intelligence, 25(2), 218-233. https://doi.org/10.1109/TPAMI.2003.1177153

[3] Zhong Zhang, Takeshi Enomoto, Tetsuo Miyake, \& Takashi Imamura. (2007). Blind source separation by combining indepandent component analysis with complex discrete wavelet transform. 2007 International Conference on Wavelet Analysis and Pattern Recognition, 549-554. https://doi.org/10.1109/ICWAPR.2007.4420731

[4] Schölkopf, B., Smola, A., \& Müller, K.-R. (1998). Nonlinear Component Analysis as a Kernel Eigenvalue Problem. Neural Computation, 10(5), 1299-1319. https://doi.org/10.1162/ 089976698300017467

[5] Bo Pang, Zhang, D., \& Kuanquan Wang. (2005). The bi-elliptical deformable contour and its application to automated tongue segmentation in Chinese medicine. IEEE Transactions on Medical Imaging, 24(8), 946-956. https://doi.org/10.1109/TMI.2005.850552

[6] Sato, Y., \& Ikeuchi, K. (n.d.). Temporal-color space analysis of reflection. Proceedings of IEEE Conference on Computer Vision and Pattern Recognition, 570-576. https://doi.org/10.1109/ CVPR.1993.341073

[7] Wolff, L. B. (n.d.). Using polarization to separate reflection components. Proceedings CVPR '89: IEEE Computer Society Conference on Computer Vision and Pattern Recognition, 363-369. https://doi.org/10.1109/CVPR.1989.37873

[8] Yoon, K., Choi, Y., \& Kweon, I. S. (2006). Fast Separation of Reflection Components using a Specularity-Invariant Image Representation. 2006 International Conference on Image Processing, 973976. https://doi.org/10.1109/ICIP.2006.312650

[9] Chaurasiya, R., \& Ganotra, D. (2021). An Overview of Learning Approaches in Reflection Removal (pp. 411-425). https://doi.org/10.1007/978-981-15-7907-3_31

[10] Farid, H., \& Adelson, E. H. (1999). Separating reflections from images by use of independent component analysis. Journal of the Optical Society of America A, 16(9), 2136. https://doi.org/10.1364/JOSAA.16.002136

[11] Naejin Kong, Yu-Wing Tai, \& Shin, J. S. (2014). A Physically-Based Approach to Reflection Separation: From Physical Modeling to Constrained Optimization. IEEE Transactions on Pattern Analysis and Machine Intelligence, 36(2), 209-221. https://doi.org/10.1109/TPAMI.2013.45 